\title{
Efecto de los iones acetato, nitrito y tiocianato en el cambio morfológico de nanoprismas triangulares a nanodiscos de plata
}

\author{
César Torres Hernández ${ }^{a}$, Alejandro Vázquez Dimas ${ }^{a}$, Israel López Hernández ${ }^{a}$ e Idalia Gómez de la \\ Fuente $^{\mathrm{a} *}$ \\ ${ }^{a}$ Universidad Autónoma de Nuevo León, Facultad de Ciencias Químicas, Laboratorio de Materiales I, Av. Universidad, Cd. \\ Universitaria 66451, San Nicolás de los Garza, Nuevo León, México. \\ *E-mail: idaliagomezmx@yahoo.com.mx
}

Recibido 11 de agosto de 2012, Aceptado 1 de septiembre de 2012

\section{Resumen}

En el presente trabajo se estudió la capacidad de los pseudohalogenuros, acetato, nitrito y tiocianato; para llevar a cabo el cambio morfológico de nanoprismas triangulares a nanodiscos de plata. Las nanoestructuras de plata resultantes fueron caracterizadas mediante espectrofotometría UV-Vis, microscopía electrónica de barrido (SEM) y microscopía electrónica de transmisión (TEM). Los pseudohalogenuros estudiados reaccionaron de manera selectiva con iones presentes en los vértices de los nanoprismas triangulares de plata provocando el truncamiento de las esquinas ocasionando un cambio en la morfología de la nanoestructura.

Palabras clave: Nanopartículas de plata, transformación morfológica, pseudohalogenuros.

\section{Introducción}

En los últimos años la evolución en los métodos de análisis instrumental ha estado enfocada en la búsqueda de técnicas que otorguen un mayor grado de sensibilidad, reproducibilidad, sencillez y aplicabilidad dentro de una gran diversidad de tipos de muestras. El fenómeno de la dispersión Raman aumentada en superficies (SERS), el cual consiste en el aumento de una señal Raman en varios órdenes de magnitud utilizando superficies de partículas metálicas a escala nanométrica, ha sido ampliamente difundido y aplicado en distintas investigaciones científicas; por ejemplo, en el área médica [1], seguridad [2], el desarrollo de materiales [3], entre otros estudios.

La elección del sustrato SERS es de gran importancia, los metales que destacan como candidatos son el oro y la plata, esto se basa en que dichos metales poseen las propiedades ópticas para manifestar una resonancia de plasmón superficial localizada en el rango del visible-infrarrojo (de 400 a $1000 \mathrm{~nm}$ ), el cual corresponde al rango de mayor interés en espectroscopia SERS. La plata ha mostrado superar al oro en varios órdenes en magnitud en el factor de aumento de la señal de espectroscopia SERS.

Dentro de las morfologías de la plata que han mostrado mejores factores de aumento en señales SERS son aquellas que tienen superficies planas y diámetros pequeños [4]. Una de las rutas planteadas para la síntesis de nanoestructuras con estas características es la síntesis de semillas de plata y su posterior fotoconversión a nanoprismas triangulares [5]. Además, los nanoprismas triangulares se presentan una reacción superficial con iones halogenuros, los cuales truncan sus vértices transformándolos en nanodiscos [4, 6, 7]. Estas últimas estructuras presentan superficies planas y diámetros pequeños favorables para espectroscopia SERS. En los reportes de estas estructuras no coinciden respecto a los diámetros obtenidos, y al tratarse de un material aplicado en el desarrollo de una técnica analítica, es fundamental asegurar la reproducibilidad de los resultados.

Una posible explicación para la diferencia de tamaños en las nanoestructuras es que las reacciones de los halogenuros con los iones plata son difíciles de controlar debido a su alta reactividad.

En 2012 Tsai y col. [8] reportaron el efecto del ion tiocianato, anión con naturaleza química similar a los halogenuros, sobre la morfología de nanoprismas triangulares de plata y su conversión a nanodiscos, obteniendo nanoestructuras más uniformes que las obtenidas utilizando iones halogenuros. De esta manera, los autores demostraron que el cambio también es factible con este pseudohalogenuro, sin embargo no explican el origen de este comportamiento. Es por eso que en este trabajo se proponen nuevas especies químicas capaces de realizar la conversión morfológica de nanoprismas triangulares de plata a nanodiscos.

\section{Parte experimental}

La síntesis de las nanoprismas triangulares de plata se realizó de acuerdo al procedimiento planteado por Xue y col. [9]. Primeramente se mezclaron $2 \mathrm{~mL}$ de $\mathrm{NaBH}_{4} 5 \mathrm{mM}, 10 \mathrm{~mL}$ de $\mathrm{AgNO}_{3}$ $5 \mathrm{mM}$ y $30 \mathrm{~mL}$ de citrato de sodio $5 \mathrm{mM}$; 
posteriormente se irradió esta dispersión de nanoesferas de plata con luz visible durante $48 \mathrm{~h}$ empleando una lámpara de vapor de sodio de alta presión, para obtener los nanoprismas triangulares por medio de una fotoconversión.

Una vez obtenidos los nanoprismas triangulares de plata se mezclaron con los pseudohalogenuros para evaluar su efecto como agentes de conversión morfológica. En el caso del ion acetato se utilizó acetato potasio $0.040,0.060$ y $0.10 \mathrm{mM}$; para los iones nitrito se prepararon soluciones de nitrito de potasio $0.020,0.030$ y $0.050 \mathrm{mM}$; y respecto a los iones tiocianato las soluciones utilizadas fueron tiocianato de potasio $1.0,2.5,10$ y $15 \mathrm{mM}$.

\section{Resultados y discusión}

La síntesis de las nanoesferas plata planteada por Xue y col. [9] produjo una dispersión de color amarillo, característico de las nanoesferas de plata. En la Figura 1 se muestra el espectro de absorción UV-Vis de la dispersión de nanoesferas de plata, en el aparece una banda de absorción dentro del rango de longitud de onda de 380 a $400 \mathrm{~nm}$, la cual corresponde a la resonancia de plasmón superficial localizado de las nanoesferas [5].

En la figura 2 se muestra una micrografía de TEM de las nanoestructuras obtenidas por reducción de iones plata con iones borohidruro, en esta micrografía se pueden observar nanoesferas con diámetros de 10 a $20 \mathrm{~nm}$.

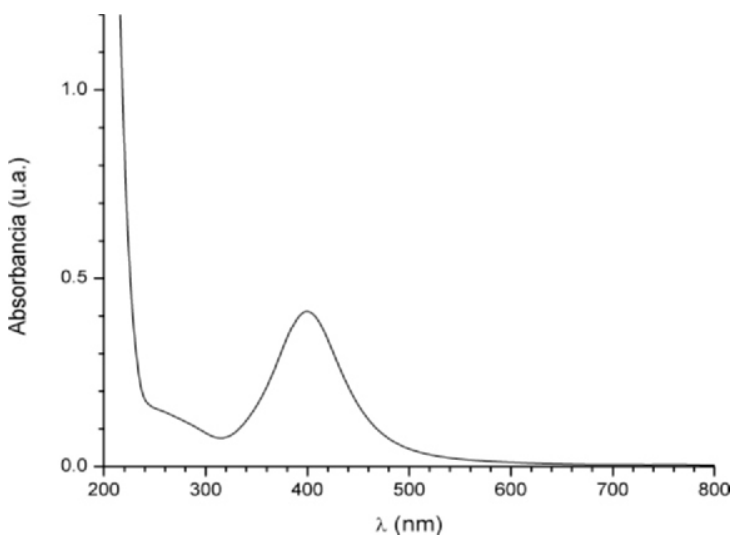

Figura 1. Espectro UV-Vis de las nanoesferas de plata.

En la figura 3 se muestra el espectro de UV-Vis de los nanoprismas triangulares de plata. En dicho espectro se puede observar una banda entre 600 y $800 \mathrm{~nm}$, teniendo una absorción máxima a los 730 $\mathrm{nm}$; además de otra banda aguda alrededor de los $330 \mathrm{~nm}$. De acuerdo con Rang y col. [10], estas bandas son asignadas a la resonancia de plasmón

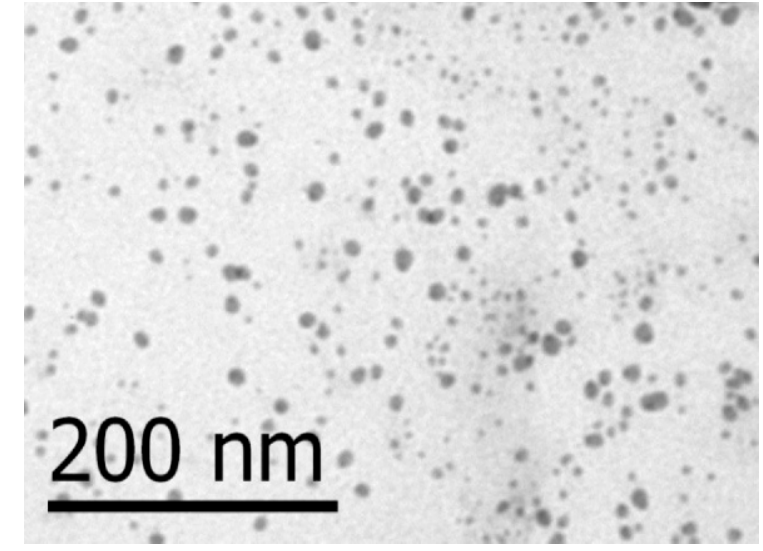

Figura 2. Imagen de TEM de las nanoesferas de plata.

dipolo en el plano y cuadrupolo fuera del plano, respectivamente; lo cual indica que la conversión de nanoesferas a nanoprismas triangulares de plata fue exitosa.

La figura 4 muestra una imagen de TEM de los nanoprismas triangulares de plata, en dicha imagen se puede apreciar que la morfología corresponde a lo predicho por medio de los espectros UV-Vis. Los nanoprismas triangulares de plata presentan aristas entre 50 y $120 \mathrm{~nm}$.

La figura 5 muestra los espectros UV-Vis correspondientes a los nanoprismas triangulares de plata en presencia de iones acetato. La desaparición gradual de las bandas características de los nanoprismas triangulares de plata indica que su concentración disminuye. Esto se puede deber a que sus vértices están siendo truncados, pero no a un grado en el cual se puedan formar los nanodiscos de plata, ya que la banda a $500 \mathrm{~nm}$, correspondientes a los nanodiscos $[4,7,8]$ está ausente. En base a lo anterior podemos concluir que no se han formado las nanoestructuras con esta morfología.

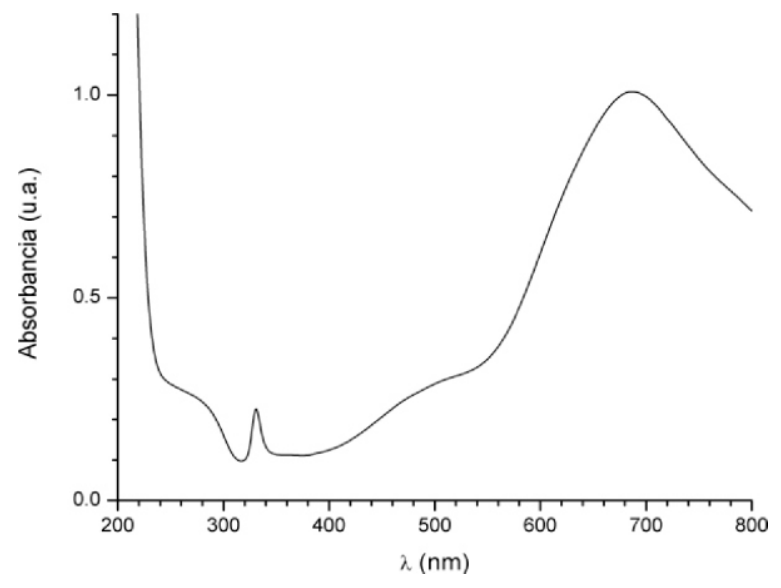

Figura 3. Espectro UV-Vis de los nanoprismas triangulares de plata 


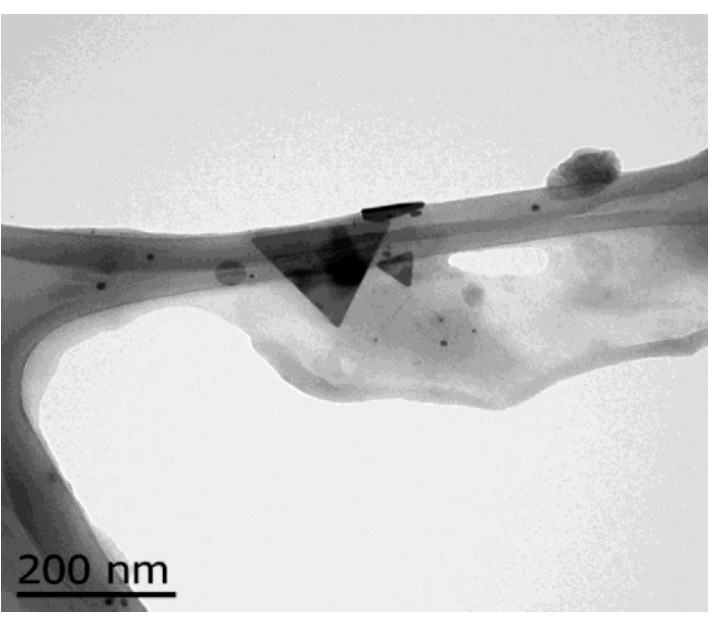

Figura 4. Imagen de TEM de los nanoprismas triangulares de plata.

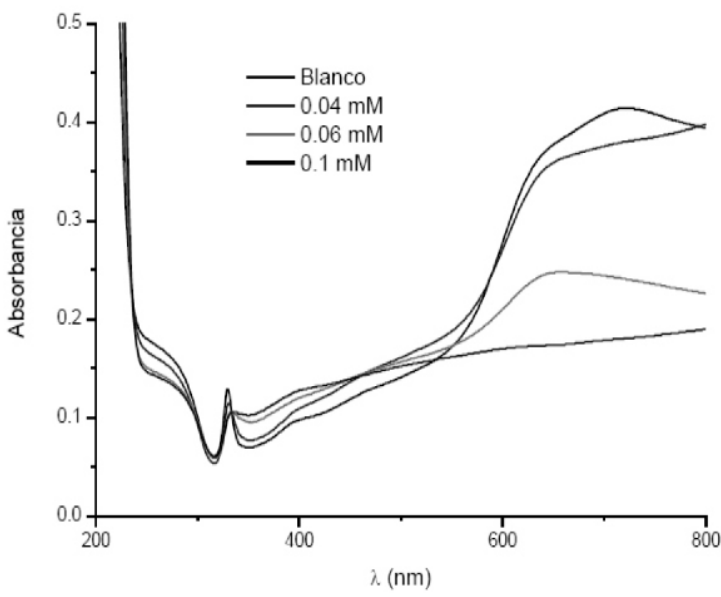

Figura 5. Espectros UV-Vis de nanoprismas triangulares de plata en presencia de iones acetato $0.040,0.060$ y 0.10 $\mathrm{mM}$.

La alteración en el espectro de los nanoprismas triangulares de plata puede deberse al cambio en las dimensiones originales de la nanoestructura y su morfología. Mediante microscopia electrónica de barrido se confirmó que el cambio morfológico de nanoprismas triangular a nanodiscos de plata, empleando el ion acetato como agente de conversión morfológica, fue negativo. En la figura 6 se puede observar que los vértices de los nanoprismas triangulares no fueron truncados en su totalidad, sin embargo se puede observar que las aristas de los nanoprismas triangulares modificaron su longitud, lo cual concuerda con lo predicho por los espectros de UV-Vis. La banda de resonancia de plasmón dipolo en el plano disminuyó su intensidad, debido a la modificación de la longitud de las aristas de los nanoprismas triangulares.

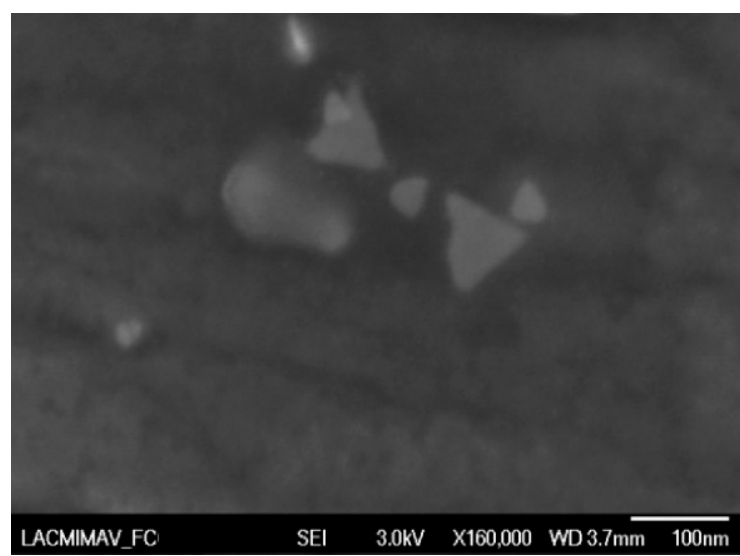

Figura 6. Imagen de SEM de los nanoprismas triangulares de plata en presencia de iones acetato.

En la figura 7 se puede observar una ligera disminución de las bandas de absorción de los nanoprismas triangulares de plata, correspondientes a los experimentos en los cuales se utilizaron iones nitrito con concentraciones 0.030 y $0.05 \mathrm{mM}$, los cuales tampoco indican truncamiento de los nanoprismas triangulares.

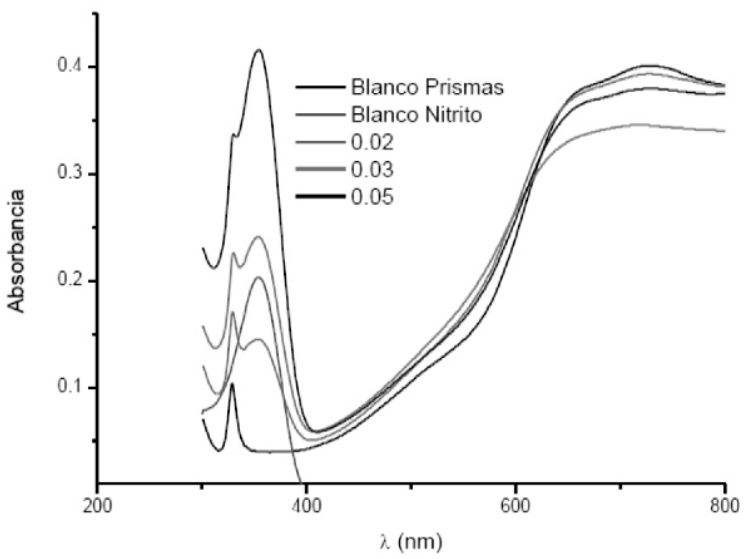

Figura 7. Espectros UV-Vis de nanoprismas triangulares de plata en presencia de iones acetato $0.020,0.030$ y 0.050 $\mathrm{mM}$.

En la figura 8 se muestra una imagen de microscopia electrónicas de barrido de los nanoprismas triangulares de plata en presencia de iones nitrito, en la cual muestra que la conversión morfológica a nanodiscos fue negativa. En la imagen se puede observar que los nanoprismas triangulares no sufrieron ningún tipo de cambio en su morfología. La variación en los espectros de UV-Vis de la figura 7 puede deberse a que los iones nitrito reaccionaron con ciertas partes de la nanoestructura, pero no significativamente. 


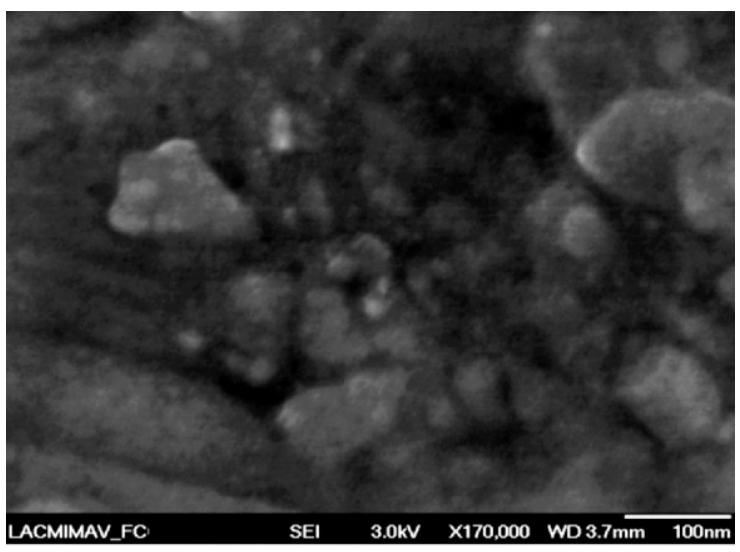

Figura 8. Imagen de SEM de los nanoprismas triangulares de plata en presencia de iones nitrito.

En la figura 9 podemos observar el espectro UVVis de nanoprismas triangulares en presencia de iones tiocianato, a la concentración $1.0 \mathrm{mM}$, en el cual se presenta la disminución de la banda de resonancia de plasmón dipolo en el plano; lo que indica que la concentración de los nanoprismas ha disminuido. Por otro lado, podemos observar que a una concentración $5 \mathrm{mM}$ la misma banda de resonancia de plasmón desapareció por completo. Lo anterior puede traducirse en que el ion tiocianato, a una concentración $5 \mathrm{mM}$, provoca una alteración morfológica significativa a los nanoprismas triangulares. Además, en el experimento a una concentración $10 \mathrm{mM}$ se puede observar que la banda que aparecía a los $460 \mathrm{~nm}$ ha sufrido un corrimiento en la longitud de onda a $485 \mathrm{~nm}$, esto nos hace pensar que la morfología y las dimensiones de las nanoestructuras de plata son más uniformes. En el experimento llevado a cabo con tiocianato 15 $\mathrm{mM}$, se observó que la dispersión tomó un color rosa claro y se presentó una banda de resonancia de plasmón a $500 \mathrm{~nm}$, la cual nos indica que la conversión de nanoprismas triangulares a nanodiscos de plata fue exitosa. Los resultados obtenidos concuerdan con lo publicado en 2012 por Tsai y col. [8], en el cual reportaron que el ion tiocianato puede llevar a cabo un cambio morfológico de nanoprismas triangulares de plata a nanodiscos.

$\mathrm{Yu}$ y col. [7] encontraron que el ion halogenuro, con el cual obtuvieron nanodiscos de menor diámetro era con el ion yoduro, sin embargo no explicaron la causa comportamiento. Por medio de una comparación con nuestros resultados podemos realizar un análisis de las constantes de los equilibrios que presentan los halogenuros con los iones plata, las cuales podrían ser uno de los factores determinantes del proceso de cambio de morfología de nanoprismas triangulares a nanodiscos.

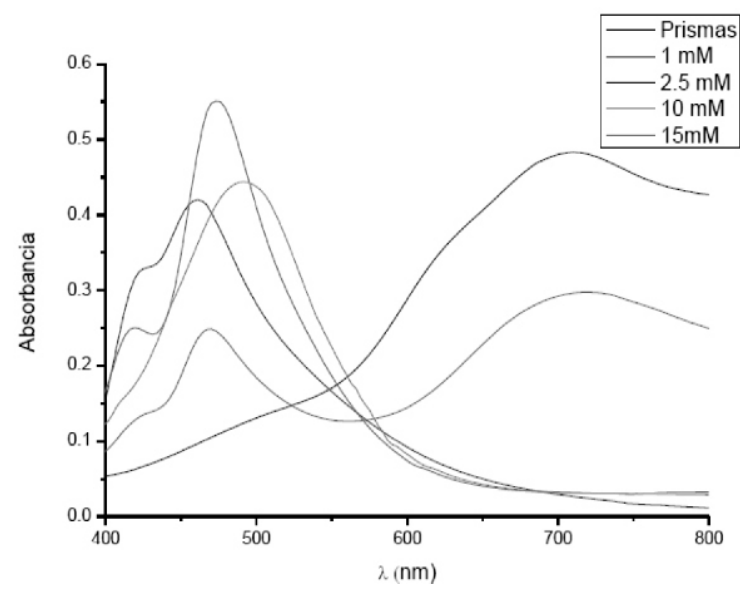

Figura 9. Espectros UV-Vis de nanoprismas triangulares de plata en presencia de iones tiocianato $1.0,2.5,10$ y 15 $\mathrm{mM}$.

Podemos observar en la Tabla 1 que el valor de la constante de producto de solubilidad de iones plata es menor con el ion yoduro. Por otro otro lado, el valor de las constantes $\beta$ de formación de complejo de iones plata es mayor con el yoduro.

Tabla 1. Valores de constantes de producto de solubilidad $\mathrm{y}$ formación de complejos de algunos halogenuros con iones plata.

\begin{tabular}{cccccc}
\hline Halogenuro & $\mathbf{p K p s}$ & $\boldsymbol{\operatorname { l o g } \boldsymbol { \beta } _ { \mathbf { 1 } }}$ & $\boldsymbol{\operatorname { l o g } \boldsymbol { \beta } _ { \mathbf { 2 } }}$ & $\boldsymbol{\operatorname { l o g } \boldsymbol { \beta } _ { \mathbf { 3 } }}$ & $\boldsymbol{\operatorname { l o g } \boldsymbol { \beta } _ { \mathbf { 4 } }}$ \\
\hline Bromuro & 12.06 & 4.15 & 7.1 & 7.95 & 8.9 \\
Cloruro & 9.5 & 3.9 & 4.7 & 5 & 5.9 \\
Yoduro & 15.83 & 13.85 & 13.7 & --- & --- \\
\hline
\end{tabular}

Realizando la misma comparación con los valores de la Tabla 2 correspondientes a los pseudohalogenuros estudiados en este trabajo, podemos observar que el ion tiocianato presenta el menor valor con respecto a la constante de producto solubilidad. Además, el valor de las constantes $\beta$ de formación de complejo de los iones plata con los pseudohalogenuros estudiados es mayor con los iones tiocianato.

Tabla 2. Valores de constantes de producto de solubilidad y formación de complejos de algunos pseudohalogenuros con iones plata.

\begin{tabular}{ccccc}
\hline Pseudohalogenuro & $\mathbf{p K p s}$ & $\log \boldsymbol{\beta}_{\mathbf{1}}$ & $\log \boldsymbol{\beta}_{\mathbf{2}}$ & $\log \boldsymbol{\beta}_{\mathbf{3}}$ \\
\hline Acetato & 2.71 & .73 & 1.37 & --- \\
Nitrito & 3.22 & --- & --- & --- \\
Tiocianato & 11.99 & 7.96 & 9.1 & 10.1 \\
\hline
\end{tabular}


En base al valor de las constantes de los equilibrios de los iones plata con los aniones estudiados en el trabajo de $\mathrm{Yu}$ y col. [7] y los resultados obtenidos en este trabajo, podemos afirmar que los iones que presentan los mejores resultados son aquellos con un valor menor de constante de producto de solubilidad y con valores de constantes $\beta$ de formación de complejo con iones plata mayores.

Los iones acetato presentan un valor mayor de constante de producto de solubilidad con iones plata respecto a los iones nitrito, sin embargo se puede observar que los iones acetato disminuyen en mayor cantidad la intensidad de la banda del plasmón de los nanoprismas triangulares de plata; lo anterior puede adjudicarse a la ausencia de equilibrios de formación de complejo de los iones nitrito con los iones plata.

\section{Conclusión}

Los pseudohalogenuros acetato y nitrito presentan una pobre efectividad para llevar a cabo la transformación morfológica de nanoprismas triangulares a nanodiscos de plata, mientras que con el ion tiocianato se logra observar una conversión total.

El comportamiento de cada uno de los iones se puede atribuir a las magnitudes de las constantes y el tipo de equilibrio que presentan con los iones plata.

\section{Referencias}

1. Yoon, K. J.; Seo, H. K.; Hwang, H.; et al. Bull. Korean Chem. Soc. 2010, 31, 1215-1218.

2. Zhang, X.; Young, M. A.; et al. J. Am. Chem. Soc. 2005, 127, 4484-4489.

3. Tao, A.; Kim, F.; et al. Nano Lett. 2003, 3, 1229-1233.

4. Ciou, S. H.; Cao, Y. W.; et al. J. Phys. Chem. C 2009, 113, 9520-9525.

5. Xue, C.; Métraux, G. S.; et al. J. Am. Chem. Soc. 2008, 130, 8337-8344.

6. An, J. ; Tang, B.; et al. J. Phys. Chem. C 2008, 112, 1517615182.

7. Yu, P.; Huang, J.; et al. J. Chin. Chem. Soc. 2010, 57, 528533.

8. Tsai, C. M.; Hsu, M. S.; et al. J. Phys. Chem. C 2012, 116, 461-467.

9. Rang, M.; Jones, A. C.; et al. Nano Lett. 2008, 8, 3357-3363. 